HUTP-93/A008, RIMS-915

\title{
Holomorphic Anomalies in Topological Field Theories
}

\author{
M. Bershadsky, S. Cecotti, 1, H. Ooguri2 and C. Vafa \\ Lyman Laboratory of Physics \\ Harvard University \\ Cambridge, MA 02138, USA
}

\begin{abstract}
We study the stringy genus one partition function of $N=2$ SCFT's. It is shown how to compute this using an anomaly in decoupling of BRST trivial states from the partition function. A particular limit of this partition function yields the partition function of topological theory coupled to topological gravity. As an application we compute the number of holomorphic elliptic curves over certain Calabi-Yau manifolds including the quintic threefold. This may be viewed as the first application of mirror symmetry at the string quantum level.
\end{abstract}

$2 / 93$

1 On leave from SISSA-ISAS and INFN sez. di Trieste, Trieste, Italy.

2 On leave from RIMS, Kyoto University, Kyoto, Japan. 
There has been much progress in understanding $N=2$ quantum field theories in two dimensions in the last few years. The main reason for this progress has to do with the appreciation of the existence of a topological subsector of these theories, represented by chiral fields ( $F$-terms in the supersymmetric terminology), giving rise to a natural ring which control many aspects of these theories. In the case of sigma models on Kähler manifolds chiral fields are in one to one correspondence with the cohomology classes of the manifold and give rise to a quantum cohomology ring. In particular the chiral fields are responsible for perturbations changing the (complexified) Kähler class of the manifold. These theories also have an 'anti-topological' sector, which is described by the CPT conjugate anti-chiral fields. In addition the theory admits an infinite number of non-topological deformations ( $D$-terms in the supersymmetric terminology). In the case of sigma models these nontopological deformations can be viewed as arbitrary variations of the metric leaving the Kähler class invariant.

There are many computations in these theories which do not depend on any of the infinite dimensional non-topological perturbations represented by the $D$-terms and depend only on the topological $(t)$ and anti-topological $(\bar{t})$ parameters representing deformations of the action using chiral and anti-chiral fields respectively (the $t$ parameters should be viewed as holomorphic coordinates parametrizing $N=2 \mathrm{QFT}$ ). This gave rise to a new notion of a 'generalized topological index' as any computation which would be independent of the non-topological deformations [1] [2]. In this sense the chiral ring (which depend only on $t$ ) and the anti-chiral ring (which depend only on $\bar{t}$ ) are the simplest examples. However there are more interesting examples of such computations depending on both $t$ and $\bar{t}$, such as the Zamolodchikov metric on moduli space. The basic tool to compute these new indices is the equations derived in [3] called $t t^{*}$ equations (topological anti-topological equations) which capture the geometry of the vacuum bundle as a function of the perturbations. These equations are a generalization of the equations representing the variations of Hodge structure for Calabi-Yau manifolds (known as special geometry) [4] to arbitrary $N=2$ QFT's.

For massive $N=2$ QFT's the $t t^{*}$ equations were shown to be the same equations as those capturing the $n$-point spin correlation of the Ising model [2] off criticality. However the spin correlations, which are given by the famous tau function, are constructed nontrivially from solution to these equations. Since the spin correlations are an intrinsic object in the Ising model the question was raised as to what is the $N=2$ analog of the tau function; in other words in purely $N=2$ terms, how do we think about the tau 
function. This was found, in [2], to be represented by a generalized index which for an $N=2$ superconformal theory can be defined as

$$
F_{1}=\int_{\mathcal{F}} \frac{d^{2} \tau}{\tau_{2}} \operatorname{Tr}(-1)^{\mathrm{F}} \mathrm{F}_{\mathrm{L}} \mathrm{F}_{\mathrm{R}} q^{\mathrm{L}_{0}} \bar{q}^{\overline{\mathrm{L}}_{0}}
$$

where the integral is over the fundamental domain $\mathcal{F}$ of moduli space of torus, $\mathrm{F}_{\mathrm{L}, \mathrm{R}}$ denote the left and right fermion numbers and the trace is over the Ramond sector for both left- and right-movers. We have to delete the contribution of the ground states of the supersymmetric Ramond sector to $F_{1}\left(L_{0}=\bar{L}_{0}=0\right)$ for the integral to converge. But since we are interested in variations of this object as a function of chiral and anti-chiral perturbations, this subtraction is irrelevant. In other words $F_{1}$ is only defined up to an addition of a constant. Some properties of this index were discussed in [2] $]^{3}$. It is our intention in this paper to further uncover some interesting properties of this index.

Let us first review the main result for this index derived in [2] (with a modification due to a subtle contact term that was missing in [2]; see Appendix A). The $N=2$ algebra implies that $F_{1}$ is essentially the sum of a holomorphic and an anti-holomorphic function of moduli, except there is a slight anomaly mixing the two expressed by

$$
\partial_{\bar{j}} \partial_{i} F_{1}=\operatorname{Tr}(-1)^{F} C_{i} \bar{C}_{j}-\frac{1}{12} G_{i \bar{j}} \operatorname{Tr}(-1)^{F}
$$

where $C_{i}$ (resp. $\bar{C}_{j}$ ) is the matrix representing the multiplication of $\phi_{i}$ (resp. $\bar{\phi}_{j}$ ) on the Ramond ground states, and $G_{i \bar{j}}$ is the Zamolodchikov metric (which appears in the above due to the contribution of a contact term discussed in appendix A). This equation is strong enough to yield $F_{1}$. The idea is that we can use the above equation to write

$$
F_{1}=\log [M(t, \bar{t}) f(t) \overline{f(t)}]
$$

where, as shown in [2] the term $M$ can be computed using (2) to be

$$
\log M=\sum_{p, q}(-1)^{p-q} \frac{p+q}{2} \operatorname{Tr}_{p, q}[\log (g)]-\frac{1}{12} K \operatorname{Tr}(-1)^{F}
$$

where $p, q$ denote the left and right Ramond charges of the vacua (which in the sigma model case on an $n$-fold range from $-n / 2, \ldots, n / 2), g$ denotes the ground state inner product in the

3 Including the relation of this index with the generalization of Ray-Singer analytic torsion to loop space. 
$p, q$ sector and $K$ is the Kähler function for the Zamolodchikov metric $(\langle\overline{0} \mid 0\rangle=\exp (-K))$. The $g$ can be computed using the $t t^{*}$ equations [3] (which in the case of sigma models on Calabi-Yau is a generalization of special geometry [4 to arbitrary $n$-fold). We are only left with holomorphic function $f(t)$ to be determined. This can in general be fixed using regularity of $F_{1}$ in the interior of moduli space and once we know how $F_{1}$ should behave at the boundaries of the moduli space 1 . This is indeed the same idea which is used in the context of computing threshold corrections for heterotic strings compactified on Calabi-Yau manifolds [5].

To give an idea how one may compute the behavior of $F_{1}$ at the boundaries of moduli space, let us consider an $N=2 \mathrm{SCFT}$ arising from a supersymmetric sigma model on a Calabi-Yau manifold $M$ of complex dimension $n$, and let us take the $t$ 's to correspond to complexified choices for the Kähler class of the manifold. To be precise we mean that the Kähler class of the manifold is taken to be

$$
k=\sum_{i}\left(t_{i}+\bar{t}_{i}\right) k_{i}
$$

where $k_{i}$ span a basis for $H^{1,1}(M, \mathbf{Z})$. Then the large $t, \bar{t} \rightarrow \infty$ of $F_{1}$ can be computed, using the fact that in this limit only constant maps dominate the path integral and the leading term comes from integrals over the zero modes of the bosonic and fermionic terms in the action. One has

$$
\begin{gathered}
\left.\operatorname{Tr}\left[(-1)^{F} \mathrm{~F}_{\mathrm{L}} \mathrm{F}_{\mathrm{R}} q^{L_{0}} \bar{q}^{\bar{L}_{0}}\right]\right|_{\infty}=\frac{1}{\left(2 \pi \tau_{2}\right)^{n}} \int d \mu\left(\prod_{r} d \psi^{\bar{r}} d \psi^{r} d \chi^{\bar{r}} d \chi^{r}\right) \times \\
\times g_{i \bar{\jmath}} \psi^{i} \psi^{\bar{\jmath}} g_{k \bar{l}} \chi^{k} \chi^{\bar{l}} \exp \left[-\tau_{2} R_{i \bar{\jmath} k \bar{l}} \psi^{i} \psi^{\bar{\jmath}} \chi^{k} \chi^{\bar{l}}\right]
\end{gathered}
$$

where $\left.\right|_{\infty}$ means the contribution in the limit $t, \bar{t} \rightarrow \infty$ which comes only from constant maps, $n$ is the complex dimension of the target space and $d \mu$ its volume form. The simplest way to get eq.(5) is to realize that the limit $t, \bar{t} \rightarrow \infty$ is just the classical theory, and then use the corresponding classical ensemble to evaluate (5) 5 . The rhs of (5) can be rewritten as

$$
\frac{(-1)^{n-1}}{(n-1) !(2 \pi)^{n} \tau_{2}} \int d \mu g_{i \bar{\jmath}} \psi^{i} \psi^{\bar{\jmath}} g_{k \bar{l}} \chi^{k} \chi^{\bar{l}}\left(R_{i \bar{\jmath} k \bar{l}} \psi^{i} \psi^{\bar{\jmath}} \chi^{k} \chi^{\bar{l}}\right)^{n-1}
$$

\footnotetext{
4 Note that (3) and (4) imply that $f(t)$ is a holomorphic section of a certain line bundle on moduli space and $F_{1}=\log \|f\|^{2}$.

${ }^{5}$ In fact, the rhs of (5) is just the classical $1 d$ computation.
} 
Integrating away the fermions we get

$$
\frac{(-1)^{n-1}}{(2 \pi)^{m}(n-1) ! \tau_{2}} \int d \mu \epsilon^{i_{1} \ldots i_{n}} \epsilon^{\bar{\imath}_{1} \ldots \bar{\imath}_{n}} \epsilon^{j_{1} \ldots j_{n}} \epsilon^{\bar{\jmath}_{1} \ldots \bar{\jmath}_{n}} g_{i_{1} \bar{\imath}_{1}} g_{j_{1} \bar{\jmath}_{1}} R_{i_{2} \bar{\imath}_{2} j_{2} \bar{\jmath}_{2}} \ldots R_{i_{n} \bar{\imath}_{n} j_{n} \bar{\jmath}_{n}} .
$$

Next, recall the formula for the $k$-th Chern class of a (complex) manifold $M$

$$
c_{k}(M)=\frac{(-1)^{k}}{(2 \pi i)^{k} k !} \delta_{i_{1} \ldots i_{k}}^{j_{1} \ldots j_{k}} R_{j_{1}}^{i_{1}} \wedge \cdots \wedge R_{j_{k}}^{i_{k}}
$$

since

$$
\epsilon^{i_{1} \ldots i_{n}} \epsilon_{i_{1} k_{1} \ldots k_{n}}=\delta_{k_{2} \ldots k_{n}}^{i_{2} \ldots i_{n}}
$$

we have the identity

$$
\begin{aligned}
& (-1)^{n-1} k \wedge c_{n-1}= \\
& =\frac{2(-1)^{n-1}}{(2 \pi)^{n}(n-1) !} d \mu \epsilon^{i_{1} \ldots i_{n}} \epsilon^{\bar{\imath}_{1} \ldots \bar{\imath}_{n}} \epsilon^{j_{1} \ldots j_{n}} \epsilon^{\bar{\jmath}_{1} \ldots \bar{\jmath}_{n}} g_{i_{1} \bar{\imath}_{1}} g_{j_{1} \bar{\jmath}_{1}} R_{i_{2} \bar{\tau}_{2} j_{2} \bar{\jmath}_{2}} \ldots R_{i_{n} \bar{\imath}_{n} j_{n} \bar{\jmath}_{n}},
\end{aligned}
$$

where $k=2 i g_{i \bar{\jmath}} d X^{i} \wedge d \bar{X}^{j}$. Using this in (5), we get

$$
\left.\operatorname{Tr}\left[(-1)^{F} \mathrm{~F}_{\mathrm{L}} \mathrm{F}_{\mathrm{R}} q^{L_{0}} \bar{q}^{\bar{L}_{0}}\right]\right|_{\infty}=\frac{(-1)^{n-1}}{(4 \pi) \tau_{2}} \int_{M} k \wedge c_{n-1}(M)
$$

Then the leading term of $F_{1}$ in the $t, \bar{t} \rightarrow \infty$ is equal to

$$
\begin{aligned}
\int_{\mathcal{F}} \frac{d^{2} \tau}{\tau_{2}} \operatorname{Tr} & {\left.\left[(-1)^{F} \mathrm{~F}_{\mathrm{L}} \mathrm{F}_{\mathrm{R}} q^{L_{0}} \bar{q}^{\bar{L}_{0}}\right]\right|_{t, \bar{t} \rightarrow \infty}=} \\
& =(-1)^{n-1} \int_{M} k \wedge c_{n-1} \int_{\mathcal{F}} \frac{d^{2} \tau}{4 \pi\left(\tau_{2}\right)^{2}}=\frac{(-1)^{n-1}}{12} \int_{M} k \wedge c_{n-1}
\end{aligned}
$$

In the special case $M$ is a (smooth) CY 3-fold one has

$$
\int k \wedge c_{2}=\frac{1}{8 \pi^{2}} \int\|R\|^{2} d \mu \geq 0,
$$

and so the leading term vanishes if and only if $M$ is flat.

Let us consider the example of a target space being a one dimensional complex torus which we denote by $T^{2}$. It is well known that the Kähler moduli of the torus is equivalent to its complex moduli and both are parametrized by the upper half plane up to the action of $\operatorname{PSl}(2, Z)$. Let us denote the Kähler class of $T^{2}$ by $\sigma$ and its complex structure by $\rho$. 
This definition of $\sigma$ differs from that discussed above by $t=-2 \pi i \sigma$. Then applying (2) to this case we see that

$$
\partial_{\sigma} \partial_{\bar{\sigma}} F_{1}=\frac{-1}{(\sigma-\bar{\sigma})^{2}}
$$

Therefore we immediately learn that

$$
F_{1}=-\log \left(\sigma_{2}|f(\sigma)|^{2}\right)
$$

where $\sigma_{2}$ is the imaginary part of $\sigma$. We are now left to compute $f(\sigma)$. However, we know that the moduli space of $\sigma$ can be taken to be the standard fundamental domain on the upper half plane, and $F_{1}$ should be a well defined function on this domain (which fixes the modular weight of $f$ ) and that for all $\sigma \neq \infty$ it should be finite. We learn therefore that

$$
F_{1}=-\log \sigma_{2}\left|\eta^{2}(q)\right|^{2}
$$

where $q=\exp (2 \pi i \sigma)$ and $\eta$ is the Dedekind eta function. Note that the leading behavior of $F_{1}$ for large $\sigma$ is also in accord with that predicted by (7) (with $n=1$, and $\int k=$ $[-2 \pi i \sigma+$ c.c. $])$.

\section{Topological Limit}

Before turning to computations for $F_{1}$, we will connect our index to the genus one partition function of topological sigma model coupled to topological gravity [6]. To this end let us consider the limit $\bar{t} \rightarrow \infty$ while fixing $t$ at a finite value. Formally this is what one would expect to be the relevant contribution for the topological theory which weighs only holomorphic maps from the world sheet to the target space. The reason $\bar{t} \rightarrow \infty$ accomplishes this is that in this limit the action is infinite unless we have a holomorphic map ( otherwise $\bar{t} \int k_{i \bar{j}} \bar{\partial} X^{i} \partial \bar{X}^{j} \rightarrow \infty$ ).

Let us first examine the case when the target space is the one dimensional complex torus. We have already computed this in (8) but it is instructive to derive this more directly by explicitly computing $\operatorname{Tr}(-1)^{F} \mathrm{~F}_{\mathrm{L}} \mathrm{F}_{\mathrm{R}} q^{L_{0}} \bar{q}^{\bar{L}_{0}}$. Since the non-zero modes of the bosons and the fermions cancel each other, it is expressed as a sum over instantons as

$$
\begin{aligned}
\operatorname{Tr}(-1)^{F} \mathrm{~F}_{\mathrm{L}} \mathrm{F}_{\mathrm{R}} q^{L_{0}} \bar{q}^{\bar{L}_{0}}=\frac{t+\bar{t}}{4 \pi \tau_{2}} \sum_{m, n, r, s} \exp \left[-\frac{t}{4 \tau_{2} \rho_{2}}|(m+r \rho)-\bar{\tau}(n+s \rho)|^{2}-\right. \\
\left.-\frac{\bar{t}}{4 \tau_{2} \rho_{2}}|(m+r \rho)-\tau(n+s \rho)|^{2}\right]
\end{aligned}
$$


where $\rho$ is the complex modulus of the target torus. In the $\bar{t} \rightarrow \infty$ limit, this becomes

$$
\begin{aligned}
& \operatorname{Tr}(-1)^{F} \mathrm{~F}_{\mathrm{L}} \mathrm{F}_{\mathrm{R}} q^{L_{0}} \bar{q}^{\bar{L}_{0}} \simeq \\
& \simeq \frac{t+\bar{t}}{4 \pi \tau_{2}}+\sum_{M \in G L(2, \mathbf{Z})} \frac{\tau_{2}}{|\operatorname{det} M|} e^{-|\operatorname{det} M| t} \delta(\tau-M(\rho))+O\left(e^{-\bar{t}}\right)
\end{aligned}
$$

where $M(\rho)=(m+r \rho) /(n+s \rho)$ for $M=\left(\begin{array}{cc}n & s \\ m & r\end{array}\right)$. The first term in the right-hand side corresponds to the zero instanton sector $(m, n, r, s=0)$ while the second term represents the sum over holomorphic instantons. In fact, when $\tau=M(\rho)$, there exists a holomorphic map of degree $|\operatorname{det} M|$ given by

$$
X(z)=(n+s \rho) z
$$

By integrating (9) over the moduli space of the worldsheet torus, we obtain 6

$$
\begin{aligned}
\frac{\partial}{\partial t} F_{1}^{t o p} & \left.\equiv \int \frac{d^{2} \tau}{\tau_{2}} \frac{\partial}{\partial t} \operatorname{Tr}(-1)^{F} \mathrm{~F}_{\mathrm{L}} \mathrm{F}_{\mathrm{R}} q^{L_{0}} \bar{q}^{\bar{L}_{0}}\right|_{\bar{t} \rightarrow \infty} \\
& =\frac{1}{12}-\sum_{M: M(\rho) \in \mathcal{F}} e^{-|\operatorname{det} M| t}
\end{aligned}
$$

Here the sum in the right-hand side is over $M \in G L(2, \mathbf{Z})$ such that $M(\rho)$ is in the fundamental domain $\mathcal{F}$ of the moduli space. Equivalently, one may sum over elements of $G L(2, \mathbf{Z})$ with the identification $M \sim U M$ for $U \in S L(2, \mathbf{Z})$. A theorem by Hermite [7] states that we can always find a canonical representative in the equivalence class of $M$ of the form

$$
\left(\begin{array}{cc}
n & 0 \\
m & \pm r
\end{array}\right), \quad n, r \geq 1, m=0, \ldots, n-1
$$

and that this representative is unique. Thus the above becomes

$$
\begin{aligned}
\frac{\partial}{\partial t} F_{1}^{t o p} & =\frac{1}{12}-2 \sum_{n, r=1}^{\infty} \sum_{m=0}^{n-1} e^{-n r t} \\
& =\frac{1}{12}-2 \sum_{n=1}^{\infty} \frac{n e^{-n t}}{1-e^{-n t}}=-2 \frac{\partial}{\partial t} \log \eta(\exp (-t))
\end{aligned}
$$

6 We are computing the derivative of $F_{1}$ with respect to $t$ since $F_{1}$ itself contains an infinite constant as mentioned before. In general, the infinity arises in $F_{1}$ when $\operatorname{Tr}(-1)^{F} \mathrm{~F}_{\mathrm{L}} \mathrm{F}_{\mathrm{R}} q^{L_{0}} \bar{q}^{L_{0}}$ does not vanish at $\tau_{2} \rightarrow 0$. 
This agrees with the expression (8) we have obtained by solving the differential equation (21) as $\bar{t} \rightarrow \infty$. It is amusing to note that this result is along the same lines as those of [8] in connecting the large $N$ expansion of pure $2 \mathrm{~d}$ QCD viewed as a string theory. Indeed the computation above is essentially the same as that in [8] but now with a reinterpretation in terms of a topological matter (represented by a target torus) coupled with topological gravity. It is tempting to conjecture that the two are indeed the same for arbitrary target spacel.

In the case of the torus, there is in fact only one primitive instanton $X(z)=z$ which appears at $\tau=\rho$, and all other instantons at $\tau=M(\rho)(M \neq 1)$ may be regarded as its multiple cover. Since the target space torus can be viewed as an infinite plane divided by translations generated by 1 and $\rho$, an image of the worldsheet should be on a fundamantal domain of a lattice defined by $n$ and $m \pm r \rho$ for some $n, r \geq 1, m=0, \ldots, n-1$ (upto the modular transformation on the target). In this case, the modulus $\tau$ of the worldsheet is given by $\tau=(m+r \rho) / n$, and the holomorphic map is $X(z)=n z$. This corresponds to the matrix $M=\left(\begin{array}{cc}n & 0 \\ m & \pm r\end{array}\right)$ and it explains why a matrix of this form gives a canonical representative of the equivalence relation. As one can see in the expansion (10), all these instantons are counted with multiplicity one in $\partial_{t} F_{1}^{t o p}$ independently of their degrees. Note also the factor of 2 in front of $\log (\eta)$ simply expresses the fact that for each holomorphic map there is another one obtained from it by sending $z \rightarrow-z$. So the moral of the story is that in the case of target space a torus there is only one primitive elliptic curve. All the other ones are multiple covers of it which just recapture the geometry of the $\eta$ function.

Let us consider the more general case when the target space is an $n$-dimensional Ricci-flat Kähler manifold $M$. To start with, let us suppose there exists a holomorphic map $X_{0}^{i}(z)$ from the worldsheet to the target at a special value of the worldsheet modulus $\tau=\tau_{0}$. In order to evaluate its contribution to the path integral in the limit $\bar{t}_{a} \rightarrow \infty$, we expand the non-linear $\sigma$ model action $S$ upto the second order in $\tau-\tau_{0}$ and $x=X-X_{0}$ as

$$
\begin{aligned}
S & =\sum_{a}\left(t_{a}+\bar{t}_{a}\left(\frac{\left|\tau-\tau_{0}\right|}{2 \tau_{2}}\right)^{2}\right) s_{a}\left(X_{0}\right)+ \\
& +\sum_{a} \bar{t}_{a}\left(\int k_{i \bar{j}}^{(a)} \partial x^{\bar{j}} \bar{\partial} x^{i}+\frac{i}{2} \psi_{L}^{i} \bar{\partial}\left(k_{i \bar{j}}^{(a)} \psi_{L}^{\bar{j}}\right)+\frac{i}{2} \psi_{R}^{\bar{j}} \bar{\partial}\left(k_{i \bar{j}}^{(a)} \psi_{R}^{i}\right)\right) d^{2} z+O\left(\frac{1}{\bar{t}}\right)
\end{aligned}
$$

7 This observation and the above counting of holomorphic maps from the torus to the torus was also made independently in [9]. 
where $s_{a}\left(X_{0}\right)=\int k_{i \bar{j}}^{(a)} \partial X_{0}^{i} \bar{\partial} X_{0}^{\bar{j}}$ are integers given by the homology class of the image. The action for $x$ and $\psi_{L}, \psi_{R}$ becomes free in this limit and is invariant under the reduced BRST transformation $\left(\delta x^{i}, \delta x^{\bar{i}}\right)=\left(i \epsilon \psi_{L}^{i}, i \bar{\epsilon} \psi_{R}^{\bar{i}}\right),\left(\delta \psi_{R}^{i}, \delta \psi_{L}^{\bar{i}}\right)=\left(2 \epsilon \partial x^{i}, 2 \bar{\epsilon} \bar{\partial} x^{\bar{i}}\right)$. This guarantees that the non-zero modes of $x$ and $\psi$ cancel as in the torus example.

When the holomorphic map $X_{0}$ corresponds to an isolated elliptic curve in $M$, there is one fermion zero mode for each of $\psi_{L}, \bar{\psi}_{L}, \psi_{R}$ and $\bar{\psi}_{R}$, and they are soaked up by the insertions of $\mathrm{F}_{\mathrm{L}}$ and $\mathrm{F}_{\mathrm{R}}$. If the map has moduli, there are additional fermion zero modes corresponding to deformations of the curve. Therefore such $X_{0}$ does not contribute to $\operatorname{tr}(-1)^{F} \mathrm{~F}_{\mathrm{L}} \mathrm{F}_{\mathrm{R}} q^{L_{0}} \bar{q}^{\bar{L}_{0}}$ to the leading order in $\bar{t}$. In the following, we only consider the situation when the curve is isolated.

Let us evaluate the zero mode integral. If the holomorphic map $X_{0}(z)$ is primitive, we can choose local coordinates near the image of $X_{0}(z)$ in the target such that the coordinate along the image coincide with that of the worldsheet. With this choice of coordinates, the zero mode integral becomes essentially two-dimensional since there is no fermion zero mode in the directions normal to the image of $X_{0}(z)$. Therefore the zero mode integral with $\mathrm{F}_{\mathrm{L}}$ and $\mathrm{F}_{\mathrm{R}}$ insertions gives the area of the image of $X_{0}(z)$ in the target space divided by $4 \pi \tau_{2}$. If the holomorphic map $X_{0}$ is primitive, the map is injective and the area is given by $\int g_{i \bar{j}} \partial X_{0}^{i} \bar{\partial} \bar{X}_{0}^{\bar{j}} d^{2} z=\sum_{a}\left(t_{a}+\bar{t}_{a}\right) s_{a}\left(X_{0}\right)$. On the other hand, if the map is an $N$-fold cover of a primitive map, it may be viewed as the composition of the primitive instanton with an $N$-fold covering of the torus by torus, discussed before. Therefore, as we saw before, we must divide this by $N$ (because of the $U(1)$ isometry of the torus).

Thus we find that, in the limit of $\bar{t}_{a} \rightarrow \infty$, the contribution of the instanton $X_{0}(z)$ to $\operatorname{tr}(-1)^{F} \mathrm{~F}_{\mathrm{L}} \mathrm{F}_{\mathrm{R}} q^{L_{0}} \bar{q}^{\bar{L}_{0}}$ is given by

$$
\begin{aligned}
& \left(\frac{\sum_{a}\left(t_{a}+\bar{t}_{a}\right) s_{a}\left(X_{0}\right)}{4 \pi \tau_{2} N}\right) e^{-\sum_{a} t_{a} s_{a}\left(X_{0}\right)}\left(\frac{4 \pi\left(\tau_{2}\right)^{2}}{\sum_{a} \bar{t}_{a} s_{a}\left(X_{0}\right)}\right) \delta\left(\tau-\tau_{0}\right) \\
& \simeq\left(\frac{\tau_{2}}{N} e^{-\sum_{a} t_{a} s_{a}\left(X_{0}\right)}\right) \delta\left(\tau-\tau_{0}\right),
\end{aligned}
$$

where the $\delta$-function (with its prefactor) emerges from taking the $\bar{t} \rightarrow \infty$ of $\exp (-S)$. Then the contribution to $F_{1}^{\text {top }}$ obtained by integrating the above integrand over the moduli space of tori with measure $d^{2} \tau / \tau_{2}$ is given by

$$
\begin{aligned}
\sum_{\begin{array}{c}
\text { elliptic } \\
\text { instantons }
\end{array}} \frac{1}{N} e^{-\sum t_{a} s_{a}} & =2 \sum_{s} d_{s} \sum_{n, r=1}^{\infty} \sum_{m=0}^{n-1} \frac{1}{n r} e^{-n r \sum_{a} t_{a} s_{a}} \\
& =-2 \sum_{s} d_{s} \log \prod_{n=1}^{\infty}\left(1-e^{-n \sum_{a} t_{a} s_{a}}\right)
\end{aligned}
$$


where the integers $s_{a}$ label the homology class of the elliptic curve and $d_{s}$ denotes the number of curves in the class.

However (11) is not the full story. Other configurations contribute to $F_{1}^{t o p}$. For instance, any meromorphic function on the torus will give us a map from the world-sheet to a sphere which, composed with a genus zero instanton, gives an instanton whose image is a rational curve in $M$. Thus $F_{1}^{t o p}$ should also get contributions from the rational curves of $M$. In fact the real story is even subtler. We also get a contribution from the rational curves in degree 1, although there is no degree 1 meromorphic function. The point is that in order to reduce the path integral to a sum over the minima of $S$ we must compactify the integration space 8 (or, equivalently, take into account the saddle points at infinity).

Let us make the simplifying assumption that we are dealing with a threefold, in which case the rational curves are (generically) rigid (to avoid integration over the moduli of rational curves). Suppose we want to construct a degree one instanton from the torus into a sphere in $M$. We can think of constructing an approximate one by 'gluing' at one point $z_{0}$ on the torus a standard instanton for the plane having a size much smaller than the periods of the torus. Of course, this is not an exact solution of the equation of motion. But it becomes so in the limit of vanishing size, where we get a delta-function instanton. Alternatively, while taking the size to zero we can make a compensating conformal transformation of a neighborhood of $z_{0}$, so that the instanton looks of finite size. In this case the world sheet will look as a sphere attached by the point $z_{0}$ to the torus, the sphere being mapped into the given rational curve of $M$ while the torus gets mapped into $X_{0}\left(z_{0}\right)$. This is the 'bubbling' phenomenon discussed in refs. [10] [11]. This phenomenon is crucial to get the correct answer for the instanton correction (even in genus zero). For a given rational curve, the leading contribution to the topological one-point function comes from the 'single bubble' configuration. It is not too hard to guess what its contribution should be. A delta-function instanton introduces a second puncture on the torus and hence one has to integrate over the corresponding moduli space, getting a factor $1 / 12\left[=\chi\left(\mathcal{M}_{1,2}\right)\right]$. The integral over the sphere then gives a factor of the degree of the corresponding rational curve. Finally, given that $F_{1}^{t o p}$ counts each elliptic curve twice (by $\mathbf{Z}_{2}$-symmetry) it is also natural to expect a similar factor here. The contribution to $\partial_{a} F_{1}^{t o p}$ from these rational

8 A convenient compactification of 'the space of all maps' was introduced by Gromov [10]. 
instantons in the threefold case is then expected to be (by a natural extrapolation of the genus zero result for the contribution of multiple cover of rational curves)

$$
-\frac{2}{12} \sum_{s} n_{s} s_{a} \frac{e^{-\sum t_{a^{\prime}} s_{a^{\prime}}}}{1-e^{-\sum t_{a^{\prime}} s_{a^{\prime}}}},
$$

where $n_{s}$ denotes the multiplicity of primitive holomorphic maps from sphere to $M$ in the given homology class. It can be shown (see appendix B by S. Katz) that (12) is the natural formula for the rational contribution to $\partial_{a} F_{1}^{t o p}$ from the viewpoint of Algebraic Geometry in the spirit of ref. 12.

Combining (11) with (12) and the contribution from the zero instanton sector which was evaluated before, we obtain

$$
\begin{aligned}
& \frac{\partial}{\partial t_{a}} F_{1}^{t o p}=\frac{(-1)^{n-1}}{12} \int k_{a} \wedge c_{n-1}- \\
& \quad-2 \sum_{s} d_{s} \sum_{n=1}^{\infty} \frac{n s_{a} e^{-n \sum t_{a^{\prime}} s_{a^{\prime}}}}{1-e^{-n \sum t_{a^{\prime}} s_{a^{\prime}}}}-\frac{2}{12} \sum_{s} n_{s} s_{a} \frac{e^{-\sum t_{a^{\prime}} s_{a^{\prime}}}}{1-e^{-\sum t_{a^{\prime}} s_{a^{\prime}}}} \\
& \quad=-2 \frac{\partial}{\partial t_{a}}\left[\sum_{s}\left[d_{s} \log \eta\left(q^{s}\right)+\frac{1}{12} n_{s} \log \left(1-q^{s}\right)\right]\right]+\text { const. }
\end{aligned}
$$

where $q^{s}=\exp \left(-t_{a} s_{a}\right)$ and $d_{s}, n_{s}$ are the number of primitive holomorphic maps from torus and sphere to $M$, respectively. The torus example is the case where $d_{s}=n_{s}=0$ for all $s$ except $d_{1}=1$.

We have thus seen that in the limit as $\bar{t} \rightarrow \infty, \partial_{i} F_{1}$ essentially counts the number of elliptic curves in the target space such that one point of the torus is mapped to the cycle dual to the Kähler class $k_{i}$. This is precisely the definition of topological gravity coupled to topological sigma model [6]. In other words in the limits as $\bar{t} \rightarrow \infty$ we can view $F_{1}$ as computing the partition function of topological gravity coupled to topological sigma model. However, we have learned more than that here: We have learned that the partition function of topological gravity coupled to topological matter has a deformation in terms of anti-chiral fields parametrized by $\bar{t}$. This is a surprise in that $\bar{t}$ perturbations are BRST trivial in the topological set up. In the topological $\sigma$-model without coupling to the gravity, it is known that the instanton approximation is exact. What we are learning here is that the story changes dramatically when we couple to topological gravity. In this case perturbing the topological theory coupled to gravity, by BRST trivial operators nevertheless modifies the partition function. In other words we have discovered that there 
is a topological anomaly in the sense that BRST trivial states fail to decouple and there is a finite boundary contribution. Indeed the equation (2) could be viewed as precisely expressing this anomaly! It is simply expressing the fact that in the definition of $F_{1}$ we have an unavoidable mixing between $t$ and $\bar{t}$. Luckily, as is the situation with anomalies, it is precisely for this reason that we are able to compute $F_{1}$ at all!

Having set up all this machinery we now apply it to some examples for low dimensions.

\section{Low Dimensional Examples and Quantum Mirror Symmetry}

After the one dimensional case, we come to the case of K3. This is a simple case as the right hand side of (2) vanishes as can be seen from (4). For $K 3$ the leading term (7) also vanishes identically since $c_{1}(K 3)=0$. Therefore $F_{1}=0$ (due to the quasi-compactness of moduli space). This is consistent with the fact that there are no holomorphic elliptic curves ${ }^{9}$ on a generic K3. Indeed, there are (non-algebraic) K3's having no non-constant meromorphic functions [13]. One shows (see [14] Th.5.1) that on such a K3 there is only a finite number of irreducible curves $C_{k}$. Moreover the sum of their genera is given by the formula

$$
\sum_{k} g\left(C_{k}\right) \leq q-p_{g}+1 \equiv 0
$$

and then $g\left(C_{k}\right)=0$ for all $k$ 's. So the first non-trivial case is a threefold CY.

Let us first write the special form (3) takes in the case of the threefold. Let us focus on the Kähler moduli (which by mirror symmetry is general enough). Let $K$ denote the Kähler function for the Zamolodchikov metric $G_{i \bar{j}}=g_{i \bar{j}} / g_{0 \overline{0}}=\partial_{i} \bar{\partial}_{j} K$ on the Kähler moduli. Then from (4) we get

$$
F_{1}=\log \left[\exp \left[\left(3+h_{1,1}-\frac{\chi}{12}\right) K\right] \operatorname{det} G_{i \bar{j}}^{-1}|f|^{2}\right]
$$

where $\chi$ is the Euler characteristic of the CY and $h_{1,1}$ is the dimensions of $H^{1,1}(M, \mathbf{Z})$ and $f$ is some holomorphic function to be determined by imposing appropriate boundary conditions at $F_{1}$ and using the fact that it should be finite in the interior of the moduli space.

The simplest non-trivial examples of threefolds are the toroidal orbifolds. The story is essentially identical to what is known for the threshold corrections [5]. In this case it is easy to see that as long as we are interested in the Kähler moduli of the underlying torus,

9 The contribution from rational curves vanishes, as it does in genus zero. 
$F_{1}$ will be independent of it in case no group element leaves a one dimensional complex torus fixed (the right hand side of (2) vanishes for such cases) or proportional to the result for one dimensional torus, in case there is a fixed torus $\left(F_{L}, F_{R}\right.$ absorb the fermion zero modes of the fixed torus) and we end up getting (in the simplest orbifolds)

$$
F_{1}=\sum c_{i} \log \left(\tau_{2}^{i} \eta^{2}\left(\tau^{i}\right) \bar{\eta}^{2}\left(\bar{\tau}^{i}\right)\right)
$$

where $c_{i}$ are some easily computable numbers, and $\tau^{i}$ refer to the Kähler moduli of the three tori of the orbifolds. Of course if we are interested in the dependence on blow up modes life is more complicated. Therefore if we ignore the blow up modes, the situation is hardly more interesting than the one dimensional case discussed before.

For a more interesting example let us consider the case of the quintic threefold. Our considerations here in fixing the holomorphic piece of $F_{1}$ are very similar to those worked out in [15] in the context of threshold corrections. Using the general form (14) and noting that $\chi=-200$ and $h_{1,1}=1$ we have

$$
F_{1}=\log \left\{G_{\psi \bar{\psi}}^{-1} \exp \left[\frac{62}{3} K\right]|f(\psi)|^{2}\right\}
$$

In this case the explicit form of $K$ is worked out in [16] using the mirror symmetry discovered for the quintic in [17] and we are using their results as well as their notation. Here we are applying this symmetry at the string one-loop level, which is thus at the quantum leve10. The modulus parameter $\psi$ describes a degenerate CY only at $\psi=1, \infty$. So regularity implies that $F_{1}$ should be finite everywhere except possibly at these two points. Moreover, multiplication of $\psi$ by a fifth root of unity is a modular transformation and should leave $F_{1}$ invariant. Finally by general arguments we know how $F_{1}$ should behave in the large volume limit, which for us is the limit $\psi \rightarrow \infty$ at which limit $\psi^{5} \sim \exp (t)$. Using (7) and the simple computation which shows

$$
\int k \wedge c_{2}=50
$$

we learn that the large $\psi$ limit of $F_{1}$ should be given by

$$
F_{1} \rightarrow \frac{50}{12}(t+\bar{t})=\frac{50}{12} \log \left|\psi^{5}\right|^{2}
$$

10 In the sense we are using the word quantum here, the quantum cohomology ring should be viewed as the classical string computation, i.e., on the sphere. 
We also need the fact that $G_{\psi \bar{\psi}}$ is regular at $\psi=0$ but $\exp [K]$ goes like $|\psi|^{-2}$ near this point and that as $\psi \rightarrow \infty, G_{\psi \bar{\psi}}^{-1}$ diverges as $|\psi|^{2}$ but $\exp [K]$ does not have a power law dependence on $\psi$ in this limit [16]. Now imposing regularity of $F_{1}$ at $\psi=0$ and the requisite divergence at $\psi=\infty(15)$ we arrive at our final result for $F_{1}$ :

$$
F_{1}=\log \left\{G_{\psi \bar{\psi}}^{-1} \exp \left[\frac{62}{3} K\right]\left|\psi^{\frac{62}{3}}\left(1-\psi^{5}\right)^{-\frac{1}{6}}\right|^{2}\right\}
$$

From what we mentioned before, this expression should contain in it the number of holomorphic maps from the torus to the quintic! All we have to do is to fix $\psi$ but take the $\bar{\psi} \rightarrow \infty$ limit. Let us discuss this in full generality for arbitrary threefold first and then apply it to the quintic as a special case.

We want to consider the behaviour of $F_{1}$ in the limit in which the 'anti-holomorphic volume' goes to infinity. The key formula is

$$
e^{-K}=\varpi^{\dagger} \Omega \varpi
$$

where $\varpi$ is the Kähler period ( $\kappa$-period) in a symplectic basis. $\varpi$ depends holomorphically on the Kähler moduli $z_{\alpha}$. In (17) $\Omega$ is the standard symplectic matrix.

If $t_{i}$ are 'good coordinates' (in the sense of special geometry), the $\kappa$-period map takes the form

$$
\varpi^{t}=\left(X_{0}, X_{i}, \partial_{0} \mathcal{F}, \partial_{i} \mathcal{F}\right)
$$

where $t_{i}=X_{i} / X_{0}$ and $\mathcal{F}$ is the Kähler prepotential. Here it is convenient to use the homogeneous coordinates $X_{I}$ because $\varpi$ takes value in some line bundle $\mathcal{L}: X_{0}$ corresponds to a choice of trivialization of $\mathcal{L}$ (i.e. by a choice of gauge we can set $X_{0}=1$ - however this is not necessarily the most convenient gauge[1]). Then the usual considerations 12 give for $t \rightarrow \infty$

$$
\mathcal{F}\left(X_{0}, X_{i}\right)=\frac{d_{i j k} X_{i} X_{j} X_{k}}{X_{0}}+c X_{0}^{2}+O\left(e^{2 \pi i t}\right),
$$

where $d_{i j k}=\int \omega_{i} \wedge \omega_{j} \wedge \omega_{k}$ is the intersection form for the $(1,1)$ forms and $c$ is some constant (generated by the loop corrections).

11 We shall always use a holomorphic gauge, i.e. $X_{0}$ is assumed to depend holomorphically on the moduli $z_{\alpha}$.

12 The analog formula in the mirror picture (i.e. if the $t$ 's are regarded as complex deformations), is known as the Schmid orbit theorems [18]. 
Given the 'factorized' form of eq.(17), taking the limit $\bar{t}_{i} \rightarrow \infty$ while keeping $t_{j}$ fixed is a well defined procedure. More precisely, we make $\bar{t}_{i} \rightarrow \bar{s}_{i}$ and send $\bar{s}$ to infinity. In this limit one has (up to exponentially small terms)

$$
\varpi^{\dagger}=\bar{X}_{0}\left(1, \bar{s} \bar{t}_{i},-\bar{s}^{3} \bar{d}_{i j k} \bar{t}_{i} \bar{t}_{j} \bar{t}_{k}+2 \bar{c}, 3 \bar{d}_{i j k} \bar{s}^{2} \bar{d}_{i j k} \bar{t}_{j} \bar{t}_{k}\right)
$$

Therefore

$$
e^{-K}=\sum_{r=0}^{3} \bar{s}^{r} A_{r},
$$

with

$$
\begin{aligned}
& A_{3}=\left|X_{0}\right|^{2} \bar{d}_{i j k} \bar{t}_{i} \bar{t}_{j} \bar{t}_{k} \\
& A_{2}=-3\left|X_{0}\right|^{2} \bar{d}_{i j k} t_{i} \bar{t}_{j} \bar{t}_{k} \\
& A_{1}=\bar{X}_{0} \bar{t}_{i} \partial_{i} \mathcal{F} \\
& A_{0}=\bar{X}_{0} \mathcal{F}-2 \bar{c}\left|X_{0}\right|^{2} .
\end{aligned}
$$

Notice that in special coordinates $A_{3}$ and $A_{2}$ take a universal form (that is, they are independent of $\mathcal{F}$ ). From (17), (18) one has

$$
K=-\log X_{0}-\log \bar{X}_{0}-\log \left[\bar{s}^{3} d_{\bar{i} \bar{j} \bar{k}} \bar{t}_{i} \bar{t}_{j} \bar{t}_{k}-3 \bar{s}^{2} d_{\bar{i} \bar{j} \bar{k}} t_{i} \bar{t}_{j} \bar{t}_{k}\right]+O\left(\bar{s}^{-2}\right),
$$

and then

$$
G_{\alpha \bar{\beta}}=\frac{1}{\bar{s}^{2}} L_{i \bar{j}}(\bar{t}) \frac{\partial t_{i}}{\partial z_{\alpha}} \frac{\partial \bar{t}_{j}}{\partial \bar{z}_{\beta}}+O\left(\bar{s}^{-3}\right)
$$

where $L_{i \bar{j}}$ is a (non-singular) anti-holomorphic matrix 13 . Then

$$
\log \operatorname{det}\left[G_{\alpha \bar{\beta}}\right]=\log \operatorname{det}\left[\partial t^{i} / \partial z^{\alpha}\right]+\operatorname{anti}-\text { holomorphic }+O\left(\bar{s}^{-1}\right) .
$$

On the other hand,

$$
K=-\log X_{0}+\text { anti }- \text { holomorphic }+O\left(\bar{s}^{-1}\right) .
$$

The $t t^{\star}$ partition function $F_{1}$ at genus one has the form (14)

$$
F_{1}=\log \left[\exp \left(3+h_{1,1}-\frac{\chi}{12}\right) K \operatorname{det}\left[G_{\alpha \bar{\beta}}\right]^{-1}|f(z)|^{2}\right]
$$

13 In fact $L_{i \bar{j}}$ is just the classical Zamolodchikov metric in which we formally set $t_{i}=0$. 
where $f(z)$ is a holomorphic section of $\mathcal{L}^{3+h_{1,1}-\frac{\chi}{12}} \otimes \mathcal{K}$. As $\bar{s} \rightarrow \infty$ the corresponding one-point function becomes

$$
\partial_{\alpha} F_{1}^{t o p}=\partial_{\alpha} \log \left[\frac{f(z)}{X_{0}^{3+h_{1,1}-\frac{\chi}{12}} \operatorname{det}(\partial t / \partial z)}\right],
$$

notice that the expression in the bracket is just $f(z)$ normalized with respect to the canonical section of $\mathcal{L}^{3+h_{1,1}-\frac{\chi}{12}} \otimes \mathcal{K}$, i.e. $X_{0}^{3+h_{1,1}-\frac{\chi}{12}} \operatorname{det}[\partial t / \partial z]$. Eq. 222) is the topological one-point function at genus one. The only non-trivial ingredient in (22) is $f(z)$.

Applying this result to the quintic case (16) we get for the topological limit

$$
F_{1}^{t o p}=\log \left[\left(\frac{\psi}{\varpi_{0}}\right)^{\frac{62}{3}}\left(1-\psi^{5}\right)^{-\frac{1}{6}} \frac{d \psi}{d t}\right]
$$

where we are using the same gauge as discussed in [16]. From what we said before (13) we have

$$
\partial_{t} F_{1}^{t o p}=\frac{50}{12}-\sum_{n, r=1}^{\infty} \frac{2 n r d_{r} q^{n r}}{\left(1-q^{n r}\right)}-\sum_{s=1}^{\infty} \frac{2 s n_{s} q^{s}}{12\left(1-q^{s}\right)}
$$

where $d_{r}$ (resp. $n_{r}$ ) is the number of primitive elliptic (resp. rational) curves of degree $r$. From (23) (24) we can read the $d_{r}$, see table 1.

\begin{tabular}{|r|r|}
\hline Degree & $d_{r}$ \\
\hline 1 & 0 \\
2 & 0 \\
3 & 609250 \\
4 & 3721431625 \\
5 & 12129909700200 \\
6 & 31147299732677250 \\
7 & 71578406022880761750 \\
8 & 154990541752957846986500 \\
9 & 324064464310279585656399500 \\
10 & 662863774391414084612496876100 \\
\hline
\end{tabular}

Table 1. \# of elliptic curves on the quintic 3 -fold

For $r \leq 3$ the $d_{r}$ can be computed directly, giving a check of our result. Indeed that $d_{1}=d_{2}=0$ follows from general facts about curves in projective space [19]. If a curve in $\mathbf{P}^{n}$ has degree 1 (resp. 2), then it is a line (resp. a plane conic) and hence rational. In 
degree 3 one has $d_{3}=n_{2}=609250$ since on a general quintic there are as many degree 2 rational curves as degree 3 elliptic ones. In fact a degree 3 elliptic curve is necessarily a plane cubic. If $C$ is such a curve lying on the quintic, the plane containing it will meet the quintic along a plane curve which is the union of $C$ with a conic. The higher values of $d_{i}$ were not known previously. Given the structure of (24), it is quite non-trivial that $d_{i}$ come out to be integer from the computation, and that can be viewed as the first non-trivial check.

We have also done a similar computation on the three examples of threefolds studied in [20] and we find the following expressions for the topological one point function in the three cases (using their notation for labelling the three models by $k$ ):

$$
\begin{array}{cc}
\frac{d F_{1}^{t o p}}{d t}=\frac{d}{d t} \log \left[\left(\frac{\psi}{\varpi_{0}}\right)^{4+\frac{204}{12}} \frac{d \psi}{d t}\left(1-\psi^{6}\right)^{-\frac{1}{6}}\right] & k=6 \\
\frac{d F_{1}^{t o p}}{d t}=\frac{d}{d t} \log \left[\left(\frac{\psi}{\varpi_{0}}\right)^{4+\frac{296}{12}} \frac{d \psi}{d t}\left(1-\psi^{8}\right)^{-\frac{1}{6}} \psi\right] & k=8 \\
\frac{d F_{1}^{t o p}}{d t}=\frac{d}{d t} \log \left[\left(\frac{\psi}{\varpi_{0}}\right)^{4+\frac{288}{12}} \frac{d \psi}{d t}\left(1-\psi^{10}\right)^{-\frac{1}{6}} \psi\right] & k=10
\end{array}
$$

and in all three cases after the genus zero subtraction, we get integral results for $d_{r}$. The results for the first few $d_{i}$ are summarized in table 2 .

\begin{tabular}{|r|r|r|r|r|r|}
\hline Model & $\chi$ & $\int c_{2} \wedge k$ & $d_{1}$ & $d_{2}$ & $d_{3}$ \\
\hline $\mathrm{k}=5$ & -200 & 50 & 0 & 0 & 609250 \\
$\mathrm{k}=6$ & -204 & 42 & 0 & 7884 & 145114704 \\
$\mathrm{k}=8$ & -296 & 44 & 0 & 41312 & 21464350592 \\
$\mathrm{k}=10$ & -288 & 34 & 280 & 207680680 & 161279120326560 \\
\hline
\end{tabular}

Table 2. \# elliptic curves on CY weighted hypersurfaces

For the low values of $r$ they agree with the known results. In particular, for $k=6$ all degree 1 curves should be 'plane' rational, and thus $d_{1}=0$. The corresponding 'plane' meets the CY space on a curve whose other component has degree 2; computing its $c_{1}$ we see that it is a torus. Hence for $k=6$ we have $d_{2}=n_{1}=7884$.

We have generalized [21] these ideas to compute higher genus partition functions $F_{g}$ of twisted $N=2$ conformal theories coupled to gravity. The story is very similar to genus one in that $F_{g}$ is essentially only a function of $t$ with a simple $\bar{t}$ dependence characterized 
by a holomorphic anomaly, relating it to lower genus partition functions. For example for the special case of three-folds the anomaly is expressed by the equation

$$
\bar{\partial}_{i} F_{g}=\bar{C}_{i j k} e^{2 K} G^{j \bar{j}} G^{k \bar{k}}\left[D_{j} D_{k} F_{g-1}+\frac{1}{2} \sum_{r} D_{j} F_{r} D_{k} F_{g-r}\right]
$$

(where $F_{g}$ is now a section of $\mathcal{L}^{2 g-2}$ and $D_{i}$ represent covariantized derivatives). This equation can be restated as a master equation for $\exp \sum_{g}\left(\lambda e^{2 K}\right)^{g-1} F_{g}$. Again it can be shown that in the topological limit $\bar{t} \rightarrow \infty, F_{g}$ counts the number of holomorphic curves of genus $g$ in $M$. Moreover one can relate the above computation to some field theoretic computation in the context of type II superstrings, thus opening the door to the exciting possibility of obtaining certain non-perturbative results for superstrings using the master equation.

We would like to thank I. Antoniadis, L. Dixon and S.T. Yau for valuable discussions. We would also like to thank S. Hosono for sharing his Mathematica program for the examples we used in this paper. We are also grateful to S. Katz for explaining the origin of the genus zero contribution (see appendix B) that we had discovered experimentally.

The research of M. B., H. O. and C. V. was supported in part by Packard fellowship and NSF grants PHY-87-14654 and PHY-89-57162. The research of S. C. was supported in part by INFN. The research of H. O. was also supported in part by Grant-in-Aid for Scientific Research on Priority Areas 231 'Infinite Analysis' from the Ministry of Education, Science and Culture of Japan. 


\section{Appendix A. Contact Term Contribution}

Let us start with the one-point function on the torus. The derivative of $F_{1}$ with respect to $t_{i}$ brings down the integral of $\left\{Q^{-},\left[\bar{Q}^{-}, \phi_{i}(z)\right]\right\}$ over the worldsheet, where $Q^{ \pm}$ $\left(\bar{Q}^{ \pm}\right)$are left-moving (right-moving) $N=2$ supercharges and $\phi_{i}$ is the chiral primary field of dimension zero associated to the parameter $t_{i}$. If we take $Q^{-}$and $\bar{Q}^{-}$around the torus, we pick up their commutator with $\mathrm{F}_{\mathrm{L}}$ and $\mathrm{F}_{\mathrm{R}}$ as

$$
\begin{aligned}
\partial_{i} F_{1} & =\int \frac{d^{2} \tau}{\tau_{2}} \operatorname{Tr}(-1)^{\mathrm{F}} \mathrm{F}_{\mathrm{L}} \mathrm{F}_{\mathrm{R}}\left(\int d^{2} z\left\{Q^{-},\left[\bar{Q}^{-}, \phi_{i}(z)\right]\right\}\right) q^{L_{0}} \bar{q}^{\bar{L}_{0}} \\
& =\int d^{2} \tau \operatorname{Tr}(-1)^{\mathrm{F}} Q^{-} \bar{Q}^{-} \phi_{i}(0) q^{L_{0}} \bar{q}^{\bar{L}_{0}} \\
& =\int d^{2} \tau \operatorname{Tr}(-1)^{\mathrm{F}}\left(\oint \frac{d u}{-2 \pi i} G^{-}(u)\right)\left(\oint \frac{d \bar{u}^{\prime}}{2 \pi i} \bar{G}^{-}\left(\bar{u}^{\prime}\right)\right) \phi_{i}(0) q^{L_{0}} \bar{q}^{\bar{L}_{0}} .
\end{aligned}
$$

In the last line, the supercharges are expressed as contour integrals of the supercurrents $G^{-}, \bar{G}^{-}$around the cycle of the torus. Since the supercurrents are (anti-) holomorphic and single-valued on the torus, we can rewrite the above as

$$
\begin{aligned}
& \partial_{i} F_{1}=\int d^{2} \tau \operatorname{Tr}\left[(-1)^{\mathrm{F}}\right.\left(\int \frac{d^{2} u}{\pi}(\bar{\partial} \xi) G^{-}(u)\right)\left(\int \frac{d^{2} u^{\prime}}{\pi}(\partial \xi) \bar{G}^{-}\left(\bar{u}^{\prime}\right)\right) \\
&\left.\times \phi_{i}(0) q^{L_{0}} \bar{q}^{\bar{L}_{0}}\right],
\end{aligned}
$$

where $\xi(z, \bar{z})=\operatorname{Im}(z) / \operatorname{Im}(\tau)$. This is a natural expression for the stringy one-point function since $G^{-}$and $\bar{G}^{-}$are to be viewed as reparametrization ghosts and $\bar{\partial} \xi$ is the Beltrami differential associated to the modulus $\tau$ of the torus.

To compute the derivative of (A.1) with respect to $\bar{t}_{j}$, we insert the integral of $\left\{Q^{+},\left[\bar{Q}^{+}, \phi_{\bar{j}}(z)\right]\right\}$ in the trace. Again, we can take $Q^{+}$and $\bar{Q}^{+}$around the torus, and pick up their commutator with $G^{-}$and $\bar{G}^{-}$.

$$
\begin{gathered}
\partial_{\bar{j}} \partial_{i} F_{1}=\int d^{2} \tau \operatorname{Tr}\left[(-1)^{\mathrm{F}}\left(\int \frac{d^{2} u}{\pi}(\bar{\partial} \xi) 2 T(u)\right)\left(\int \frac{d^{2} u^{\prime}}{\pi}(\partial \xi) 2 \bar{T}\left(\bar{u}^{\prime}\right)\right)\right. \\
\left.\times\left(\int d^{2} z \phi_{\bar{j}}(z)\right) \phi_{i}(0) q^{L_{0}} \bar{q}^{\bar{L}_{0}}\right]
\end{gathered}
$$

By using the Ward identity on the torus [22], we can replace the energy-momentum tensor $T$ by derivatives with respect to $\tau$ and $z$ as

$$
\begin{gathered}
\partial_{\bar{j}} \partial_{i} F_{1}=4 \int d^{2} \tau \int d^{2} z\left(\partial_{\bar{\tau}}+\xi \partial_{\bar{z}}+\left(\partial_{\bar{z}} \xi\right)\right)\left(\partial_{\tau}+\xi \partial_{z}+\left(\partial_{z} \xi\right)\right) \\
\times \operatorname{Tr}(-1)^{\mathrm{F}} \phi_{\bar{j}}(z) \phi_{i}(0) q^{L_{0}} \bar{q}^{\bar{L}_{0}} .
\end{gathered}
$$


If the integrand were regular in $z$, we could interchange the integral and the derivatives in the above as $\int d^{2} z\left(\partial_{\bar{\tau}}+\xi \partial_{\bar{z}}+\left(\partial_{z} \xi\right)\right) \rightarrow \partial_{\bar{\tau}} \int d^{2} z$. However there is a singularity at $z=0$ due to the OPE of $\phi_{\bar{j}}(z)$ and $\phi_{i}(0)$ as

$$
\phi_{\bar{j}}(z) \phi_{i}(0) \sim \frac{G_{i \bar{j}}}{(2 \pi)^{2} z \bar{z}}
$$

and an additional contribution arises from the contact term

$$
\bar{\partial}\left[\xi \partial\left(\xi \phi_{\bar{j}}(z)\right)\right] \phi_{i}(0) \sim-\frac{G_{i \bar{j}}}{16 \pi\left(\tau_{2}\right)^{2}} \delta(z)+\cdots .
$$

We then obtain

$$
\begin{aligned}
\partial_{\bar{j}} \partial_{i} F_{1}= & 4 \int d^{2} \tau \frac{\partial}{\partial \bar{\tau}} \int d^{2} z\left(\partial_{\tau}+\xi \partial_{z}+\left(\partial_{z} \xi\right)\right) \operatorname{Tr}(-1)^{\mathrm{F}} \phi_{\bar{j}}(z) \phi_{i}(0) q^{L_{0}} \bar{q}^{\bar{L}_{0}}- \\
& -\int \frac{d^{2} \tau}{4 \pi\left(\tau_{2}\right)^{2}} G_{i \bar{j}} \operatorname{Tr}(-1)^{\mathrm{F}} q^{L_{0}} \bar{q}^{\bar{L}_{0}} .
\end{aligned}
$$

The second term in the right-hand side becomes $-\frac{1}{12} G_{i \bar{j}} \operatorname{Tr}(-1)^{\mathrm{F}}$ after integration over $\tau$.

Because of the $\bar{\tau}$-derivative, the first term in the right-hand side of (A.2) can be expressed as an integral at $\tau_{2} \rightarrow \infty$ as

$$
\oint_{-\frac{1}{2}+i \infty}^{\frac{1}{2}+i \infty} d \tau \int d^{2} z 2 i\left(\partial_{\tau}+\xi \partial_{z}+\left(\partial_{z} \xi\right)\right) \operatorname{Tr}(-1)^{\mathrm{F}} \phi_{\bar{j}}(z) \phi_{i}(0) q^{L_{0}} \bar{q}^{\bar{L}_{0}}
$$

The piece involving $\partial_{\tau}$ can be evaluated using the technique of [2] as

$$
\begin{aligned}
& \oint_{-\frac{1}{2}+i \infty}^{\frac{1}{2}+i \infty} d \tau \int d^{2} z 2 i \partial_{\tau} \operatorname{Tr}(-1)^{\mathrm{F}} \phi_{\bar{j}}(z) \phi_{i}(0) q^{L_{0}} \bar{q}^{\bar{L}_{0}}= \\
& =\operatorname{Tr}(-1)^{\mathrm{F}}\left(\oint d z \phi_{\bar{j}}(z)\right) P \phi_{i}(0)(P-1),
\end{aligned}
$$

where $P$ is the projection operator on the ground states. The rest is also simplified as 14

$$
\begin{aligned}
& \oint_{-\frac{1}{2}+i \infty}^{\frac{1}{2}+i \infty} d \tau \int d^{2} z 2 i\left(\partial_{z} \xi+\left(\partial_{z} \xi\right)\right) \operatorname{Tr}(-1)^{\mathrm{F}} \phi_{\bar{j}}(z) \phi_{i}(0) q^{L_{0}} \bar{q}^{\bar{L}_{0}}= \\
& =\operatorname{Tr}(-1)^{\mathrm{F}} \oint d z \phi_{\bar{j}}(z) P \phi_{i}(0) .
\end{aligned}
$$

14 The singularity at $z=0$ again generates a contact term here. But, this time, it does not survive the $\tau_{2} \rightarrow \infty$ limit. 
Putting them together, the contribution from $\tau_{2} \rightarrow \infty$ is

$$
\begin{aligned}
& \operatorname{Tr}(-1)^{\mathrm{F}}\left(\oint d z \phi_{\bar{j}}(z)\right) P \phi_{i}(0)(P-1)+\operatorname{Tr}(-1)^{\mathrm{F}}\left(\oint d z \phi_{\bar{j}}(z)\right) P \phi_{i}(0) P \\
& =\operatorname{Tr}(-1)^{\mathrm{F}}\left(\oint d z \phi_{\bar{j}}(z)\right) P \phi_{i}(0) P \\
& =\operatorname{Tr}(-1)^{\mathrm{F}} C_{i} \bar{C}_{j} .
\end{aligned}
$$

Thus we find that the holomorphic anomaly is expressed as

$$
\partial_{\bar{j}} \partial_{i} F_{1}=\operatorname{Tr}(-1)^{\mathrm{F}} C_{i} \bar{C}_{j}-\frac{1}{12} G_{i \bar{j}} \operatorname{Tr}(-1)^{\mathrm{F}}
$$

The second term in the right-hand side, which comes from the contact term between $\phi_{i}$ and $\phi_{\bar{j}}$, was missing in the previous paper [2].

\section{Appendix B. Intersection Theory over Moduli Spaces of Degenerate Instantons}

by Sheldon Katz 15

The computation of $n$ point functions via mirror manifolds has led to predictions for the number of curves of certain types on Calabi-Yau manifolds $X$. The resulting finite numbers are independent of the complex structure of $X$. It is possible for $X$ to actually contain infinitely many curves of the type in question for some complex structures and finitely many curves for others. This has led to the creation of an algebro-geometric method for calculating what the number would be if it were finite, even if the calculation took place using the "wrong" complex structure [23]. This number will be called the contribution of the family of curves in question. Evidence is emerging that even if there are infinitely many curves of the type considered for any complex structure on $X$, the number obtained by algebraic geometry coincides with the number obtained by an asymptotic expansion of the $n$ point function [12] [24]. In this appendix, we give another example of this phenomenon.

Here are the main points which have arisen in the algebro-geometric investigation.

1. We must consider degenerate instantons. This is necessary because the requirement of invariance of the number under variation of complex structure forces integration over

15 Department of Mathematics, Oklahoma State University, Stillwater, OK 74078; katz@math.okstate.edu. 
compact sets (to prevent curves from "going off to infinity" as the complex structure parameters of $X$ vary). Including degenerate instantons will always compactify the space of instantons.

2. Deformation theory tells one how to deform a holomorphic map $f: C \rightarrow X$. The tangent space to the space of all such maps is the space of global sections $T^{0}=H^{0}\left(C, f^{*} T_{X}\right)$, where $T_{X}$ is the holomorphic tangent bundle of $X$. There is an analytic map $\rho_{f}: U \rightarrow T^{1}$ from a neighborhood $U$ of the origin in $T^{0}$ to another finite dimensional vector space $T^{1}$. The vector space $T^{1}$ is called the obstruction space, and $\rho_{f}$ is the obstruction mapping. The actual space of holomorphic maps is locally described as $\rho_{f}^{-1}(0)$. In this local description, the origin of $T^{0}$ corresponds to the map $f$ itself.

3. As $f$ varies, the varying $T^{1}$ spaces form an obstruction bundle $\mathcal{T}^{1}$ (possibly with singularities) over the space of all maps. As the complex structure of $X$ is infinitesimally altered, there arises an obstruction section $\rho$ of $\mathcal{T}^{1}$, whose zero locus is precisely the locus of maps which can be deformed so as to remain holomorphic after the change in complex structure. From now on, $C$ will be a complex curve. If the dimension of the zero locus of $\rho$ is what one expects (in the present context, this means 3 if $C$ has genus 0,1 if $C$ has genus 1 , or 0 if $C$ has genus $g>1$ ), then its homology class may be calculated via the use of Chern classes. In this way, we can effectively calculate the contribution of curves on $X$ by essentially doing the computation on an infinitesimally nearby complex structure. It may be that the obstruction sections never yield the expected dimensions for any complex structure. This is the situation for example if the mappings are $d$ to 1 covers of a curve in $X$, for $d>1$. However, the Chern class may always be calculated; and it will always have the correct dimension. In such an instance, one assumes that this Chern class represents what the homology class "should be" if there indeed were a deformation of complex structure general enough to yield finitely many curves, and then proves under various assumptions that this is invariant under deformations. By analogy with excess intersection theory [25], this class is called the equivalence of the family of curves in question [23].

4. In calculating the $n$ point function, we usually count the number of instantons for which the images of the $n$ points lie in various hypersurfaces determined by the $(1,1)$ forms under consideration. Here, there is an analogous condition for all (possibly degenerate) instantons. The condition is reinterpreted as a cohomology class, which can be intersected with the result of the Chern class calculation in the last step to give a number, the contribution of the family. 
We want to apply these ideas to calculate the equivalence of the family of degenerate instantons corresponding to maps from an elliptic curve to a rational curve.

Now, for specifics. Motivated by the computation in [12], one identifies $f: C \rightarrow X$ with its graph $\Gamma_{f} \subset C \times X$. It is well known in algebraic geometry how to compactify the space of all subvarieties of a given algebraic variety (here $C \times X$ ): one uses the Hilbert scheme $H=\operatorname{Hilb}(C \times X)$ which parametrizes all subvarieties of $C \times X$ [26]. Here, we restrict our attention to the connected component of $H$ which contains $\left\{\Gamma_{f}\right\}$. Assume $\operatorname{Im}(f)=D \subset X$ is a curve which is rigid in $X$. Then all deformations of $f$ will continue to map inside $D$, and we may as well consider $\operatorname{Hilb}(C \times D)$. Let $\pi_{C}$ and $\pi_{D}$ be the projection mappings of $C \times D$, and let $c \in C$ and $d \in D$ be general points. Then if $f: C \rightarrow D$ is a degree $k$ covering, we have $\pi_{C}^{-1}(c) \cdot \Gamma_{f}=1$ and $\pi_{D}^{-1}(d) \cdot \Gamma_{f}=k$. Also, $\Gamma_{f}$ has the same genus as $C$. So we compactify by including all subvarieties $\Gamma$ of $C \times D$ such that $\Gamma \cdot \pi_{C}^{-1}(c)=1, \Gamma \cdot \pi_{D}^{-1}(d)=k$, and $g(\Gamma)=g(C)$.

Now, let $C$ be an elliptic curve, and let $D \subset X$ be a rigid rational curve, fixed for the remainder of this appendix. There are no degree 1 maps from $C \rightarrow D$. But there are still singular subvarieties of $C \times D$ as above that must be considered as degenerate instantons. Let $p \in C$ and $q \in D$ denote arbitrary points. Let $\Gamma_{p q}=(\{p\} \times D) \cup(C \times\{q\}) \subset C \times D$. Then $\Gamma_{p q}$ has genus 1 , and $\Gamma_{p q} \cdot \pi_{C}^{-1}(c)=1, \Gamma_{p q} \cdot \pi_{D}^{-1}(d)=1$. It is easy to see that these are the only possible degenerate instantons.

Before we calculate the obstruction bundle, we must identify the moduli space of degenerate instantons globally. First of all, we must not only specify an elliptic curve $C$ and points $c \in C, d \in D$, but also a marked point $p_{1} \in C$, since we need to consider pointed elliptic curves for the 1 point function. It is well known that there is no consistent way to describe what one might think of as $\{(E, p) \mid p \in E\}$. This is because of the presence of automorphisms. Given any elliptic curve $E$, the group of automorphisms acts transitively; hence modulo automorphisms, any point of $E$ is the same as any other point. However, a point cannot be selected from $E$ in a continuous fashion as $E$ varies due to the presence of extra automorphisms that certain elliptic curves possess. This situation is usually remedied by introducing a "level $k$ " structure on $E$ for some $k \geq 3$. Concretely for $k=3$, this means we consider the hypersurface $\mathcal{C}$ in $\mathbf{P}^{1} \times \mathbf{P}^{2}$ defined by the equation

$$
s\left(x^{3}+y^{3}+z^{3}\right)+t x y z=0 .
$$


Here $(s: t)$ are homogeneous coordinates for $\mathbf{P}^{1}$, and $(x: y: z)$ are homogeneous coordinates for $\mathbf{P}^{2}$. Via the projection map $\pi: \mathcal{C} \rightarrow \mathbf{P}^{1}$, this is thought of as a family of plane cubic curves parametrized by $(s, t) \in \mathbf{P}^{1}$. Each curve in the family $\mathcal{C}$ contains the marked point $p_{1}=(1,-1,0)$; so we have succeeded in giving a family of pointed elliptic curves. The problem is that we have described all elliptic curves multiply; consideration of the $j$ invariant of (B.1) shows that each elliptic curve occurs 12 times in this family (including multiplicity). So the number which results from using $\mathcal{C}$ for computational purposes must be divided by 12 at the end.

We are now ready to compute the moduli space of degenerate instantons. We need to specify a pointed elliptic curve $\left(E, p_{1}\right)$, a point $p \in E$, and a point $q \in D$. The data $\left(E, p_{1}, p\right)$ is just the specification of a point of $\mathcal{C}$ (modulo the 12 to 1 identifications given by the $j$ invariant, which will not be mentioned again until the last step). So the moduli space is in this instance just $\mathcal{C} \times D$. Note that this space is nonsingular of dimension 3 . A 1 dimensional class (the equivalence) must be obtained before imposing the condition on $p_{1}$; hence we will need to calculate a second Chern class.

The cohomology ring of $\mathcal{C} \times D$ may be easily worked out. By the Künneth formula, the result is just the tensor product of the cohomology rings of $\mathcal{C}$ and $D$. We write $h$ for the positive integral generator of $H^{2}(D) . \mathcal{C}$ is well known to be isomorphic to the blow up of $\mathbf{P}^{2}$ at the base locus of the pencil of elliptic curves defining $\mathcal{C}$, i.e. the nine points $x^{3}+y^{3}+z^{3}=x y z=0$. So $H^{2}(\mathcal{C})$ is generated by classes $H, E_{1}, \ldots, E_{9}$, where $H$ is the pullback of the hyperplane class of $\mathbf{P}^{2}$ to $\mathcal{C}$ and the $E_{i}$ are the classes of the exceptional divisors. We order the $E_{i}$ so that $E_{1}$ corresponds to the marked point $p_{1}$. Note that the pullback via $\pi$ of the hyperplane class $a$ of $\mathbf{P}^{1}$ is just $3 H-\sum_{i=1}^{9} E_{i}$.

Putting the preceding together, we see that the instantons which we consider here are identified with certain subvarieties of $C \times D$, as $C$ varies over all pointed elliptic curves. This is what is meant by the relative Hilbert scheme $\operatorname{Hilb}\left(\mathcal{C} \times D / \mathbf{P}^{1}\right)$ [26]. We specialize now to $k=1$. We have seen that the degenerate instantons are of the form $\Gamma_{p q}=(\{p\} \times D) \cup(C \times\{q\})$, where $p \in C$ and $q \in D$ and are parametrized by $\mathcal{C} \times D$. We want to describe $\Gamma_{p q}$ globally.

Consider the space $\mathcal{C} \times{ }_{\mathbf{P}^{1}} \mathcal{C} \times D \times D$. There are diagonals $\Delta_{\mathcal{C}} \subset \mathcal{C} \times{ }_{\mathbf{P}^{1}} \mathcal{C}$ and $\Delta_{D} \subset$ $D \times D$. Let $\Gamma=\left(\Delta_{\mathcal{C}} \times D \times D\right) \cup\left(\mathcal{C} \times \mathbf{P}^{1} \mathcal{C} \times \Delta_{D}\right)$. Let $\pi_{1}, \pi_{2}$ be the projections onto the respective $\mathcal{C}$ factors, and let $\rho_{1}, \rho_{2}$ be the projections onto the respective $D$ factors. Then if $C$ is a curve of the family $\mathcal{C}$, and if $p \in \mathcal{C}$ and $q \in D$, we have $\Gamma \cap\left(\pi_{1} \times \rho_{1}\right)^{-1}\{(p, q)\}=\Gamma_{p q}$. So $\Gamma$ is the "universal degenerate instanton" parametrized by $\mathcal{C} \times D$. 
Note that while $\mathcal{C}$ is smooth, the fibers of $\mathcal{C} / \mathbf{P}^{1}$ can be singular. In fact, we easily compute from (B.1) that there are 3 singular points over $s=0$, and one singular point over each of the three points with $t / s=-3 e^{2 \pi i m / 3}$, for $m=0,1,2$. Thus $\mathcal{C} \times_{\mathbf{P}^{1}} \mathcal{C}$ itself has singularities - there are 9 singular points over $s=0$, and one singular point over each point $t / s=-3 e^{2 \pi i m / 3}$, for $m=0,1,2$ [27]. Although the presence of singularities can often complicate the method of [23], we will see presently that they cause no problem in this case.

In general terms, the obstruction space $T^{1}$ at $\Gamma_{p q}$ is computed in terms of the normal sheaf of $\Gamma_{p q}$ in $C \times X$ [26] [24]. Note that for the graph $\Gamma_{f}$ of an actual holomorphic mapping $f: C \rightarrow X$, the normal sheaf of $\Gamma_{f}$ in $C \times X$ is just $f^{*}\left(T_{X}\right)$. This should be compared with [12]. The normal sheaf of a degenerate instanton may differ from the pulled back tangent bundle considered in [12]; however, when the present obstruction analysis is applied to the calculation of the contribution to the 3 point function as in [12], the identical result is obtained.

The normal sheaf roughly speaking has two parts: the normal sheaf of $\Gamma_{p q}$ in $C \times D$, and the pullback to $\Gamma_{p q}$ via $\rho_{2}$ of the normal bundle of $D$ in $X$. Note that since we have assumed that $D$ is rigid, we know that the normal bundle of $D$ in $X$ is $\mathcal{O}_{D}(-h) \oplus \mathcal{O}_{D}(-h)$.

There are no relevant obstructions associated to the normal sheaf of $\Gamma_{p q}$ in $C \times D$. We can describe the deformations of $\Gamma_{p q}$ by deforming $p$ in $C$ and $q$ in $D$. There are no obstructions to deforming $q$, and there are no obstructions to deforming $p$ unless $p$ is a singular point of a singular fiber of $\mathcal{C}$. But even in this case, the only obstructions are the obstructed tangent directions at the singular point; but these are of no concern to us.

So the only relevant obstructions come from the cohomology sheaf of the pullback of the sheaf $\mathcal{O}_{D}(-h) \oplus \mathcal{O}_{D}(-h)$. We may restrict attention to one of these factors; and we must calculate the sheaf $R^{1}\left(\left.\left(\pi_{1} \times \rho_{1}\right)\right|_{\Gamma}\right)_{*}\left(\rho_{2}^{*} \mathcal{O}_{D}(-h)\right)$ on $\mathcal{C} \times D$. We check first that this is a rank 1 bundle on $\mathcal{C} \times D$, and then that it is equal to $\mathcal{O}_{\mathcal{C} \times \mathcal{D}}(-a-h)$, where we have simplified notation by supressing the pullbacks of $a$ and $h$.

We first note that $\operatorname{dim} H^{1}\left(\Gamma_{p q}, \rho_{2}^{*} \mathcal{O}_{D}(-h)\right)=1$ for all $p$ and $q$, even for $p$ a singular point of a singular fiber. In fact, the restriction of $\rho_{2}^{*} \mathcal{O}_{D}(-h)$ to $C \times\{q\}$ is trivial, so that $\operatorname{dim} H^{1}\left(C \times\{q\}, \rho_{2}^{*} \mathcal{O}_{D}(-h)\right)=1$. We also see that the restriction map is an isomorphism by considering the exact cohomology sequence associated to the short exact sequence

$$
0 \rightarrow \mathcal{O}_{D}(-2 h) \rightarrow \rho_{2}^{*} \mathcal{O}_{D}(-h) \rightarrow \mathcal{O}_{C} \rightarrow 0,
$$


where the right hand map is restriction. The sheaf on the left arises as the subsheaf of $\mathcal{O}_{D}(-h)$ consisting of sections vanishing at $\{q\}$. We have simplified notation by using $C$ and $D$ to stand for $C \times\{q\}$ and $\{p\} \times D$, respectively.

This shows that $R^{1}\left(\left.\left(\pi_{1} \times \rho_{1}\right)\right|_{\Gamma}\right)_{*}\left(\rho_{2}^{*} \mathcal{O}_{D}(-h)\right)$ is a line bundle on $\mathcal{C} \times D$ [28] (whose fiber over $\{(p, q)\}$ is $\left.H^{1}\left(\Gamma_{p q}, \rho_{2}^{*} \mathcal{O}_{D}(-h)\right)\right)$.

The above argument also shows that

$$
R^{1}\left(\left.\left(\pi_{1} \times \rho_{1}\right)\right|_{\Gamma}\right)_{*} \rho_{2}^{*} \mathcal{O}_{D}(-h) \simeq R^{1}\left(\left(\pi_{1} \times \rho_{1}\right) \mid \mathcal{C} \times_{\mathbf{P}^{1}} \mathcal{C} \times \Delta_{D}\right)_{*} \rho_{2}^{*} \mathcal{O}_{D}(-h)
$$

since we have just shown that this isomorphism holds fiber by fiber.

Since we have restricted $D \times D$ to $\Delta_{D}$, we may replace $\rho_{2}$ by $\rho_{1}$ in $(\mathbb{B} .2)$ and compute instead

$$
R^{1}\left(\left.\left(\pi_{1} \times \rho_{1}\right)\right|_{\mathcal{C} \times \mathbf{P}^{1}} \mathcal{C} \times \Delta_{D}\right) * \rho_{1}^{*} \mathcal{O}_{D}(-h) \simeq R^{1} \pi_{1 *}\left(\mathcal{O}_{\mathcal{C} \times{ }_{\mathbf{P}^{1}} \mathcal{C} \times \Delta_{D}}\right) \otimes \pi_{D}^{*} \mathcal{O}_{D}(-h)
$$

by the projection formula. Since higher direct images commute with base extension in this instance [28], the desired result will follow from $(\overline{B .2})$ and $(\bar{B} .3)$ once we show that $R^{1} \pi_{*} \mathcal{O}_{\mathcal{C}} \simeq \mathcal{O}_{\mathbf{P}^{1}}(-a)$. This follows for example by first noting that $\operatorname{dim} H^{1}\left(\mathcal{O}_{C}\right)=1$ for all fibers $C$ of $\pi$, showing that $R^{1} \pi_{*} \mathcal{O}_{\mathcal{C}}$ is a line bundle on $\mathbf{P}^{1}$. To identify the line bundle, one can apply the Grothendieck-Riemann-Roch formula [25]. The straightforward calculation has been checked using "schubert" 29.

So the obstruction bundle $\mathcal{T}^{1}$ is just $\mathcal{O}(-a-h) \oplus \mathcal{O}(-a-h)$. Its second Chern class is $(-a-h)^{2}=2 a h$. This class is the equivalence of the family of instantons.

We next turn to the "1 point condition". That is, we choose a hyperplane section $P$ of $X$ which is a representative of the $(1,1)$ class that we are interested in 16 , and look at the set of all $\Gamma$ which contain a point of $\left\{p_{1}\right\} \times P$. This is a restatement in terms of graphs of the usual condition $f\left(p_{1}\right) \in P$. If $D \subset X$ has degree $s$ (relative to our $(1,1)$ class), then $D$ intersects $P$ in $s$ points. Let $q$ be one of these points. Then we just have to look at the set of all $\Gamma$ which contain the point $\left(p_{1}, q\right)$, and multiply this class (independent of $q$ ) by $s$. Before multiplying, this subvariety is clearly just $\left(E_{1} \times D\right) \cup(\mathcal{C} \times\{q\})$. Its class is just $E_{1}+h$.

16 Notation in this appendix differs slightly from notation in the paper. A single $(1,1)$ class is considered here for notational simplicity, rather than effectively considering all classes simultaneously by grouping curves by their homology classes rather than their degrees. 
We finally impose the 1 point condition on the equivalence. Recalling that $\pi^{*}(a)=$ $3 H-\sum_{i=1}^{9} E_{i}$, we get the following contribution from the family of degree 1 degenerate maps from the fibers of $\mathcal{C}$ to $D$.

$$
\begin{aligned}
2 a h\left(E_{1}+h\right) & =2\left(3 H-\sum_{i=1}^{9} E_{i}\right) h\left(E_{1}+h\right) \\
& =-2 E_{1}^{2} h \\
& =2
\end{aligned}
$$

Putting this all together, we get $\frac{2}{12} s n_{s}$ as the contribution of the family of degenerate maps with covering degree 1 from elliptic curves to all degree $s$ rational curves. This gives a contribution of $\frac{2}{12} s n_{s} e^{-t s}$ to the 1 point function. Here $n_{s}$ is the number of rational curves of degree $s$ in $X$, all assumed rigid.

The calculation of the contribution of degree $k$ covers of a rational curve is more complicated, since the space of degree $k$ instantons is singular. But the above analysis makes it clear that the result will be of the form $\frac{a_{k}}{12} s n_{s}$ for some integers $a_{k}$, independent of the choice of Calabi-Yau manifold. This yields the formula

$$
\frac{1}{12} \sum_{k} s n_{s} a_{k} e^{-t k s}
$$

for the contribution to the 1 point function of all (possibly degenerate) maps from elliptic curves to rational curves. We have shown that $a_{1}=2$. This analysis is consistent with (12); in the language of this paragraph, (12) asserts that $a_{k}=2$ for all $k$, but this has not yet been checked by algebraic geometry - the obstruction analysis needed in the general case is more delicate.

We would like to thank D.R. Morrison for numerous conversations relating to degenerate instantons. We would also like to thank A. Yukie for a helpful discussion about the universal level 3 elliptic curve. 


\section{References}

[1] S. Cecotti, P. Fendley, K. Intriligator and C. Vafa, Nucl. Phys. B386 (1992) 405.

[2] S. Cecotti and C. Vafa, Ising Model and N=2 Supersymmetric Theories, preprints Harvard HUTP-92/A044 and SISSA-167/92/EP (1992).

[3] S. Cecotti and C. Vafa, Nucl. Phys. B367 (1991) 359.

[4] S. Ferrara and A. Strominger, in String '92, ed. R. Arnowitt et al. (World Scientific, Singapore 1990), p.245;

S. Cecotti, Commun. Math. Phys. 131 (1990) 517;

A. Strominger, Commun. Math. Phys. 133 (1990) 163;

P. Candelas and X.C. de la Ossa, Nucl. Phys. B355 (1991) 455;

R. D'Auria, L. Castellani and S. Ferrara, Class. Quant. Grav. 1 (1990) 1767.

[5] L.J. Dixon, V.S. Kaplunovsky and J. Louis, Nucl. Phys. B355 (1991) 649;

S.Ferrara, C.Kounnas, D.Lüst and F.Zwirner, Nucl. Phys. B365 (1991) 431;

I. Antoniadis, E. Gava and K.S. Narain, preprints IC/92/50 and IC/92/51;

J.-P. Derendinger, S. Ferrara, C. Kounnas and F. Zwirner, Nucl. Phys. B372 (1992) 145 .

[6] E. Witten, Nucl. Phys. B340 (1990) 281.

[7] M. Newman, Integral Matrices, Pure and Applied Mathematics, vol 45, Academic Press, New York 1972.

[8] D. Gross and W. Taylor, Two Dimensional QCD is a String Theory, hep-th@xxx.lanl.gov 9301068 .

[9] R. Dijkgraaf, R. Rudd, in preparation.

[10] M. Gromov, Invent. Math. 82 (1985) 307.

[11] J.G. Wolfson, J. Diff. Geom. 28 (1988) 383.

[12] P.S. Aspinwall and D.R. Morrison, Topological Field Theory and Rational Curves, Oxford preprint OUTP-91-32p, DUK-M-91-12 (1991).

[13] K. Kodaira, Amer. J. Math. 87 (1964) 751.

[14] K. Kodaira, Ann. Math. 74 (1961) 591.

[15] L. Dixon, Talk Presented in Mirror Symmetry Conference at MSRI, 1991, unpublished.

[16] P. Candelas, X.C. de la Ossa, P.S. Green and L. Parkes, Nucl. Phys. B359 (1991) 21.

[17] B.R. Greene and M.R. Plesser, Nucl. Phys. B338 (1990) 15.

[18] W. Schmid, Invent. Math. 22 (1973) 211.

[19] P. Griffiths and J. Harris, Principles of Algebraic Geometry, Wiley-Interscience, New York, 1978.

[20] A. Klemm and S. Theisen, Considerations of One-Modulus Calabi-Yau Compactifications: Picard-Fuchs Equations, Kähler Potentials and Mirror Maps, preprints KATHEP-03/92, TUM-TH-143-92 (April 1992).

[21] M. Bershadsky, S. Cecotti, H. Ooguri and C. Vafa, to appear. 
[22] T. Eguchi and H. Ooguri, Nucl. Phys. B282 (1987) 308.

[23] S. Katz, Excess Intersection and Deformations, in preparation.

[24] S. Katz, in Essays on Mirror Manifolds, edited by S.-T. Yau, International Press, Hong Kong 1992.

[25] W. Fulton, Intersection Theory, Springer-Verlag, Berlin Heidelberg New York 1984.

[26] A. Grothedieck, Fondements de la Géometrie Algébrique, Séminaire Bourbaki, Secrétariat Math., Paris 1962.

[27] C. Schoen, Math. Z. 197 (1988) 177.

[28] R. Hartshorne, Algebraic Geometry, Springer-Verlag, New York Berlin Heidelberg 1977.

[29] S. Katz and S. A. Strømme, "schubert": a Maple package for intersection theory, Available by anonymous ftp from ftp.math.okstate.edu or linus.mi.uib.no, cd pub/schubert. 